\title{
Microelectromechanical System-Based Electrochemical Seismometers with Two Pairs of Electrodes Integrated on One Chip
}

\author{
Xichen Zheng ${ }^{1,2}$, Deyong Chen ${ }^{1,2, *}$, Junbo Wang ${ }^{1,2}$, Jian Chen ${ }^{1,2} \oplus$, Chao Xu ${ }^{1,2}$, Wenjie $\mathrm{Qi}^{1,2}$ \\ and Bowen Liu ${ }^{1,2}$ \\ 1 School of Electronic, Electrical and Communication Engineering, University of Chinese Academy of Sciences, \\ Beijing 100049, China; zhengxichen17@mails.ucas.ac.cn (X.Z.); jbwang@mail.ie.ac.cn (J.W.); \\ chenjian@mail.ie.ac.cn (J.C.); xuchao16@mails.ucas.edu.cn (C.X.); qiwenjie16@mails.ucas.edu.cn (W.Q.); \\ liubowen17@mails.ucas.edu.cn (B.L.) \\ 2 State Key Laboratory of Transducer Technology, Institute of Electronics, Chinese Academy of Sciences, \\ Beijing 100010, China \\ * Correspondence: dychen@mail.ie.ac.cn; Tel.: +86-136-6105-3471
}

Received: 10 August 2019; Accepted: 11 September 2019; Published: 13 September 2019

\begin{abstract}
This paper presents microelectromechanical system (MEMS)-based electrochemical seismometers with two pairs of electrodes integrated on one chip. Both theoretical analysis and numerical simulations were conducted to reveal the working principle of the proposed electrochemical seismometers, finding that flow holes distributed on cathodes rather than anodes can produce significantly higher sensitivities. The proposed electrochemical seismometers were fabricated based on conventional micromachined processes with high fabrication repeatability. Sensitivity measurements of the proposed seismometers and their commercial counterpart of CME6011 were conducted, indicating the sensitivities of the proposed seismometer with flow holes on cathodes were two orders higher than the counterpart with flow holes on anodes and one order higher than CME6011 at dominant frequencies. Measurements of random ground motions based on the proposed seismometer with flow holes on cathodes and CME6011 were conducted, producing comparable noise levels without observable ground motions and high correlations in response to random events of ground motions. These results validated the functionality of the proposed electrochemical seismometers, which may contribute to seismic monitoring in the near future.
\end{abstract}

Keywords: microelectromechanical system; electrochemical seismometer; integrated sensing unit; high sensitivity; high fabrication repeatability

\section{Introduction}

A seismometer is a core element in seismic monitoring and geological prospecting [1-3]. According to the detecting mechanisms, seismometers can be divided into moving-coil seismometers, piezoelectric seismometers, electromagnetic seismometers, capacitive seismometers, optic-fiber seismometers, and electrochemical seismometers [4-10]. Table 1 lists the advantages and disadvantages of several kinds of mainstream seismometers. Among these, electrochemical seismometers are featured with no mechanical noises, high sensitivities, and large working dip. More importantly, no mass centering and lock is needed in electrochemical seismometers as the sensing unit is immersed in liquid, and thus, compared to the other sorts of seismometers, electrochemical seismometers are more suitable for ocean-bottom seismic monitoring in complex underwater environments [11].

The sensing units of traditional electrochemical seismometers were manufactured by conventional net-waving technologies, suffering from key problems of poor consistency, low yield, and high 
cost [12-14]. To address these issues, MEMS based sensing units of electrochemical seismometers were developed by both Chen et al. and Huang et al. $[15,16]$. Chen et al. proposed a sensing unit stacked up with layers of microfabricated electrodes and insulators to improve device consistency, which, however, required accurate alignments of different layers [15]. Subsequently, Huang et al. proposed a sensing unit with silicon nitride and platinum deposited on both sides of a silicon wafer in turn, realizing high-efficiency alignment. However, the device sensitivity was relatively low due to the limited electrode area, and the manufacturing process was time consuming because of focused ion beam etching [16]. To obtain high sensitivities, Deng et al. from our group developed a sensing unit with an anode and a cathode integrated on a single chip with much larger electrode areas [17]. Nevertheless, as a troublesome step, manual alignments were required in the assembly of sensing units, which were prone to chip damage. A new method based on parylene for manufacturing sensing units without manual alignments was then put forward, in which the device sensitivity was improved by decreasing the distances between electrodes to several micrometers by our group [18]. However, the parylene-based sensor was impractical as a result of poor adhesion between the parylene and the electrode layers.

Table 1. The comparison of several kinds of mainstream seismometers.

\begin{tabular}{ccc}
\hline Types & Advantages & Disadvantages \\
\hline Moving-Coil & $\begin{array}{c}\text { Passive, } \\
\text { Low cost, } \\
\text { Simple structure }\end{array}$ & Poor low-frequency performance \\
\hline Optic-Fiber & High sensitivity & $\begin{array}{c}\text { High cost, } \\
\text { Capacitive }\end{array}$ \\
Electrochemical & High mechanical noise \\
\hline wide dynamic range & $\begin{array}{c}\text { Fragile, } \\
\text { No mechanical noises, } \\
\text { High sensitivity, } \\
\text { large wrking dip }\end{array}$ & Small working dip \\
\hline
\end{tabular}

To address the aforementioned problems, this paper presents new micromachined electrochemical seismometers with two pairs of sensing electrodes integrated on a single chip. In comparison to previously reported counterparts, the seismometers developed in this study are featured with high fabrication repeatability and high sensitivity because of enlarged electrode areas.

\section{Device Structure and Working Principle}

As shown in Figure 1a, the MEMS-based electrochemical seismometers consist of two elastic membranes, a frame, two stainless steel rings, a spring, a plexiglass shell, electrolyte, and a sensing unit. Besides, the sensing unit is immersed in an electrolyte solution of tri-iodide and iodine ions (low-amount addition of iodine to potassium iodide solution), which is encapsulated in the plexiglass shell sealed by two elastic membranes. Furthermore, as shown in Figure $1 b, c$, the sensing unit includes two pairs of electrodes in symmetry, which are separated by insulating layers and integrated on one silicon chip. In addition, numerous through holes perpendicularly to the electrode surfaces function as flow ways, which are distributed on cathodes or anodes, respectively.

In this design, the cathodes were distributed on surfaces of the silicon wafer and the side walls of flow holes, whose electrode areas were more than 500 times larger than those of previous electrochemical seismometers based on single silicon wafers, where the areas of the cathodes were defined by side walls of flow holes [16]. Therefore, electrode areas of cathodes can be effectively enlarged, leading to significant improvements in device sensitivities. In addition, the inclusion of a pair of anode and cathode integrated on a plane could simplify the fabrication process without the steps of depositing extra insulating layers. 


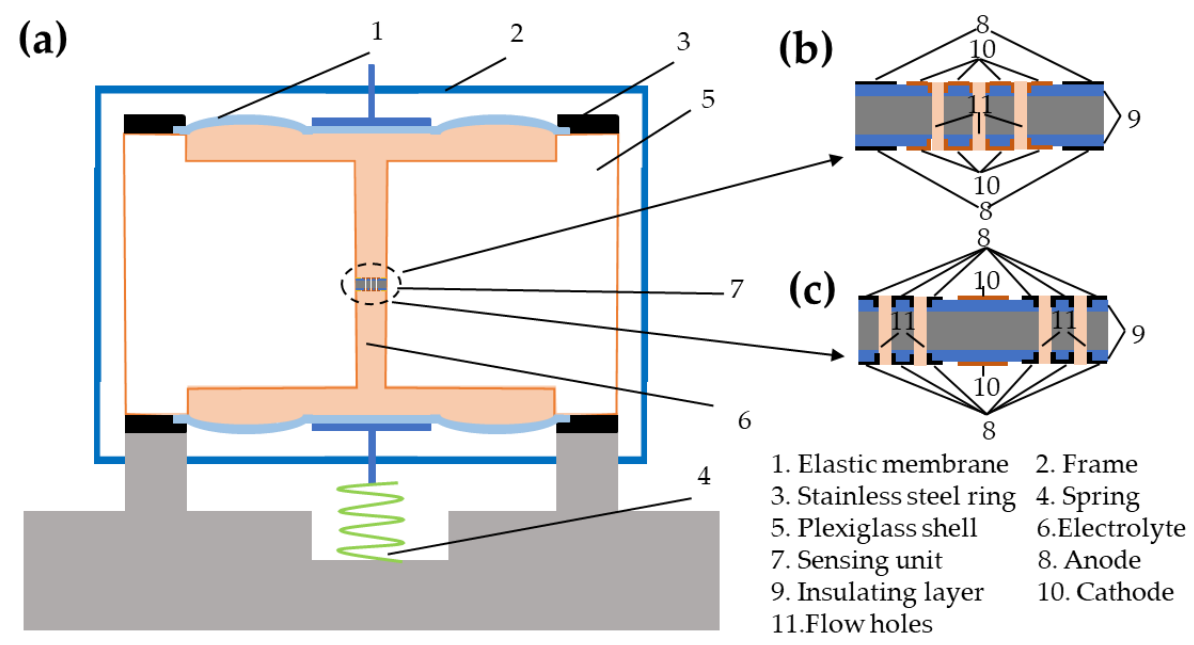

Figure 1. (a) Schematic of the MEMS based electrochemical seismometer, consisting of (1) two elastic membranes, (2) a frame, (3) two stainless steel rings, (4) a spring, (5) a plexiglass shell, (6) electrolyte, and (7) a sensing unit. The sensing unit is immersed in the electrolyte, which is encapsulated in the plexiglass shell sealed by two elastic membranes. The enlarged schematic of the sensing unit with (8) anodes, (9) insulting layers (10) cathodes, and (11) flow holes integrated on a single chip where flow holes are distributed on cathodes $(\mathbf{b})$ or anodes $(\mathbf{c})$, respectively.

When a voltage is applied to the two electrodes on the same side of the sensing unit, there are reversible electrochemical reactions occurring around the anodes and the cathodes, which are $3 I^{-}-2 \mathrm{e}^{-} \rightarrow I_{3}^{-}$and $I_{3}^{-}+2 \mathrm{e}^{-} \rightarrow 3 I^{-}$, respectively. When no vibration is applied to the seismometer, the concentration distribution of the ions on both sides is symmetrical, and thus the output voltage equates to zero. However, in case of vibrations, the ions flow relative to the sensing unit due to inertia, which results in an increase in concentration of tri-iodide ions around one cathode and a decrease in concentration of tri-iodide ions around the other cathode, generating a differential output current, with the amplitude and frequency indicating vibration status.

At first, owing to inertia, the velocity of external vibrations is converted to electrolyte velocity relative to electrodes, which then was converted to output voltage by electrochemical reaction. In fact, the electrolyte in seismometers can be considered as incompressible liquid in laminar flow. Therefore, we used Continuity equation and Navier-Stokes Equation to describe the velocity field:

$$
\begin{gathered}
\rho \nabla \vec{V}=0 \\
\frac{\partial \vec{V}}{\partial t}=-\frac{\nabla \vec{P}}{\rho}+\frac{\mu}{\rho} \nabla^{2} \vec{V}+\vec{g}
\end{gathered}
$$

where $\vec{V}$ is the velocity in the specified direction, $\vec{P}$ is the pressure, $\rho$ is the density of the electrolyte, and $\vec{g}$ is the gravity acceleration. It is obvious the distribution of reaction ions will be affected by flow of the electrolyte, diffusion, and electromigration. Thus, the ion flux $(\vec{N})$ can be expressed by Nernst-Plank Equation as following:

$$
\overrightarrow{\mathrm{N}}=-\mathrm{D} \nabla C-\frac{z F}{R T} D C \nabla \varphi+C \vec{V}
$$

The right side of the equation corresponds to flux changes caused by diffusion, electromigration, and convection, respectively. $\mathrm{D}$ is the diffusion coefficient, $\mathrm{C}$ is ion concentration, $\mathrm{z}$ is the charge of ions, $\mathrm{F}$ is the Faraday constant, $\mathrm{R}$ is the gas constant, $\mathrm{T}$ is the Calvin temperature, and $\varphi$ is the potential. In addition, the electromigration component is very small as a result of the shielding effect of potassium 
ions, and the diffusion is the main component. At the same time, there is no flux at the boundary of channels. At the electrode boundary, the reactive ion flux satisfies the Bulter-Volmer Equation:

$$
2 \vec{n} \cdot \vec{N}_{I_{3}^{-}}=-\frac{2 \vec{n} \cdot \vec{N}_{I^{-}}}{3}=-2 K_{a} C_{I_{3}^{-}} e^{-\frac{\alpha n F}{R T} *\left(U-E^{0}-\varphi\right)}+K_{c} C_{I^{-}} e^{\frac{(1-\alpha) n F}{R T} *\left(U-E^{0}-\varphi\right)}
$$

in which $\vec{n}$ is a unit normal vector of electrode surface; $\mathrm{K}_{\mathrm{a}}$ and $\mathrm{K}_{\mathrm{c}}$ represent the rate constants of cathode and anode reactions, respectively; $\alpha$ is the charge transfer coefficient for the cathodic reaction; $U$ is the imposed electrical potential between the electrodes; and $E^{0}$ is the equilibrium electrical potential. The relationship between output current on cathode and ion flux can be represented by the following Equation:

$$
\mathrm{I}=\mathrm{nF} \int_{S} \vec{n} \cdot \vec{N}_{I_{3}^{-}} d s
$$

where $\mathrm{n}$ is the number of charge in single electrode reaction and equals to one, and $\mathrm{S}$ represents the area of cathode. The final output $I_{0}$ is given by:S

$$
U_{o}=\left(I_{1}-I_{2}\right) * R
$$

where $I_{1}$ and $I_{2}$ represents the output current of two cathodes, $\mathrm{R}$ represents voltage-current conversion resistance. Combining formulas mentioned above and based on known electrolyte velocity, $U_{0}$ can be calculated.

\section{Simulation}

The electrochemical seismometers proposed in this study involved complex electrochemical analysis, and thus, finite element simulation (COMSOL Multiphysics 3.5, Stockholm, Sweden) was used for performance evaluation and optimization. As shown in Figure 2a, a two-dimensional model, consisting of the sensing unit with two pairs of electrodes integrated on one chip, two elastic membranes and electrolyte, coupled with (1) the physical field of solid mechanics, (2) the laminar flow field, and (3) the electric analysis field, was used to study the qualitative effect of key geometrical parameters of the sensing unit on the performance of the seismometers. Figure $2 b, c$ shows the sensing units with two pairs of electrodes integrated on one chip, where the flow holes were distributed on cathodes or anodes with the same areas, respectively. Taking the model with flow holes distributed on cathodes as an example, the employed key geometrical parameters were as follows (see Figure 2d): the insulating space between anode and cathode $\left(L_{1}\right)$ is $100 \mu \mathrm{m}$; the insulating space between the two cathodes $\left(L_{2}\right)$ is $130 \mu \mathrm{m}$, which also represents the length of the flow holes; and the diameter of the flow holes $\left(\mathrm{L}_{3}\right)$ is $100 \mu \mathrm{m}$. Initial concentrations of potassium iodide and iodine were set as $4000 \mathrm{~mol} / \mathrm{m}^{3}$ and $40 \mathrm{~mol} / \mathrm{m}^{3}$, respectively. The inputting volume force (time piecewise function from 0.01 to $100 \mathrm{~Hz}$ ) applied to the electrolyte was equivalent to the external vibration, which drove the liquid mass to flow.

In the simulation model of seismometers with two pairs of electrodes integrated on one chip, the electrolyte was considered as incompressible liquid in laminar flow, and the solid walls were in a no-slip boundary condition. Therefore, the physical fields of solid mechanics and laminar flow were described by the Continuity equation and the Navier-Stokes equation, respectively. At the same time, the electric analysis field was represented by Faraday's law, the Bulter-Volmer condition and the Nernst-Plank equation under the condition of no flux at the channels' boundary, where key parameters were given as follows: the charge transfer coefficient for the cathodic reaction is 0.5 , the equilibrium electrical potential is $0.54 \mathrm{~V}$, the diffusion coefficient of $\mathrm{I}_{3}^{-}$is $5.76 \times 10^{-10} \mathrm{~m}^{2} / \mathrm{s}$, and the diffusion coefficient of $\mathrm{I}^{-}$is $1.285 \times 10^{-9} \mathrm{~m}^{2} / \mathrm{s}$.

Figure 2e shows the simulation results of amplitude-frequency responses of proposed seismometers with two pairs of electrodes integrated on one chip in the frequency domain of $0.01-100 \mathrm{~Hz}$, where the flow holes were distributed on anodes or cathodes, respectively. In addition, the 
voltage-current conversion resistor is set to $1 \mathrm{~K} \Omega$. The amplitudes for the electrochemical seismometers with holes on anodes vs. cathodes were quantified as $40.24 \mathrm{~V} /(\mathrm{m} / \mathrm{s})$ vs. $278.47 \mathrm{~V} /(\mathrm{m} / \mathrm{s})$ at $0.1 \mathrm{~Hz}$, $177.58 \mathrm{~V} /(\mathrm{m} / \mathrm{s})$ vs. $3173.02 \mathrm{~V} /(\mathrm{m} / \mathrm{s})$ at $1 \mathrm{~Hz}$, and $94.46 \mathrm{~V} /(\mathrm{m} / \mathrm{s})$ vs. $419.84 \mathrm{~V} /(\mathrm{m} / \mathrm{s})$ at $10 \mathrm{~Hz}$, respectively. It was observed that the output amplitudes of the electrochemical seismometers increased firstly and then decreased with the increase of the frequency of the input volume force. This was because at the low-frequency domain the conversion efficiency between external vibrations and electrolyte movement was relatively low, while the rates of the electrochemical reactions on the electrodes were limited at the high-frequency domain. Furthermore, it was located that the output amplitudes of the seismometer with flow holes distributed on cathodes roughly one order higher than the counterpart with flow holes on anodes, owing to the fact that the concentration of tri-iodide ions around two cathodes was much higher than the counterparts with holes on anodes.

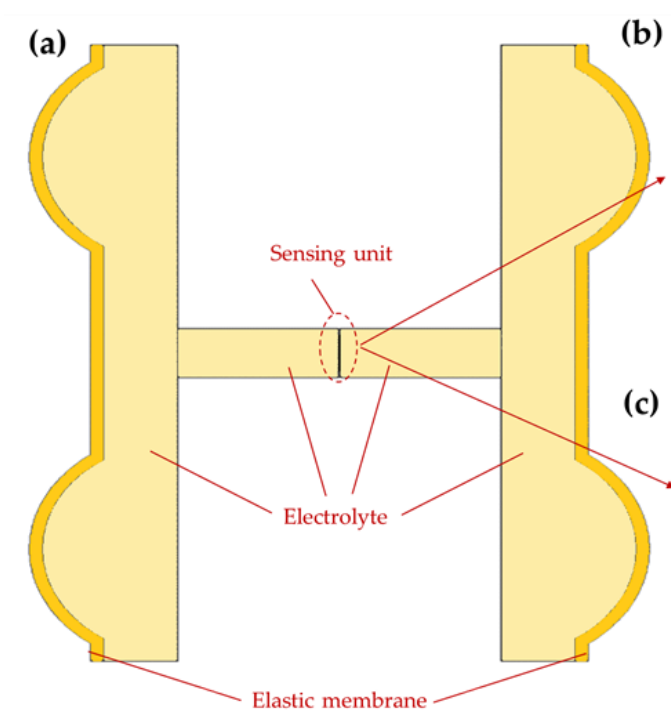

(b)

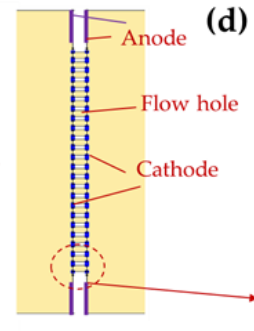

(d)

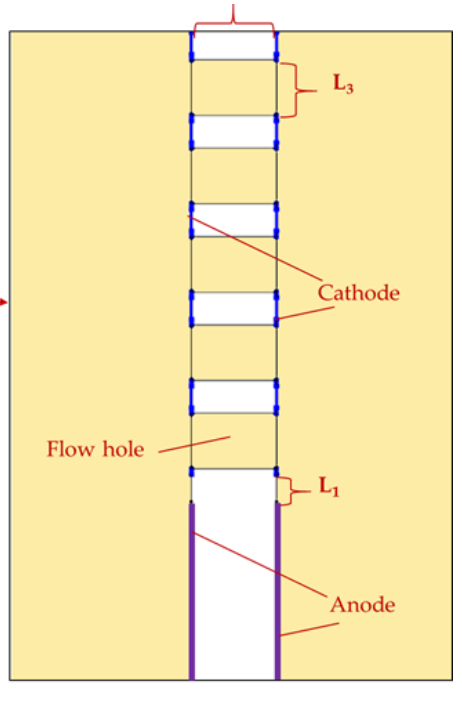

(e)

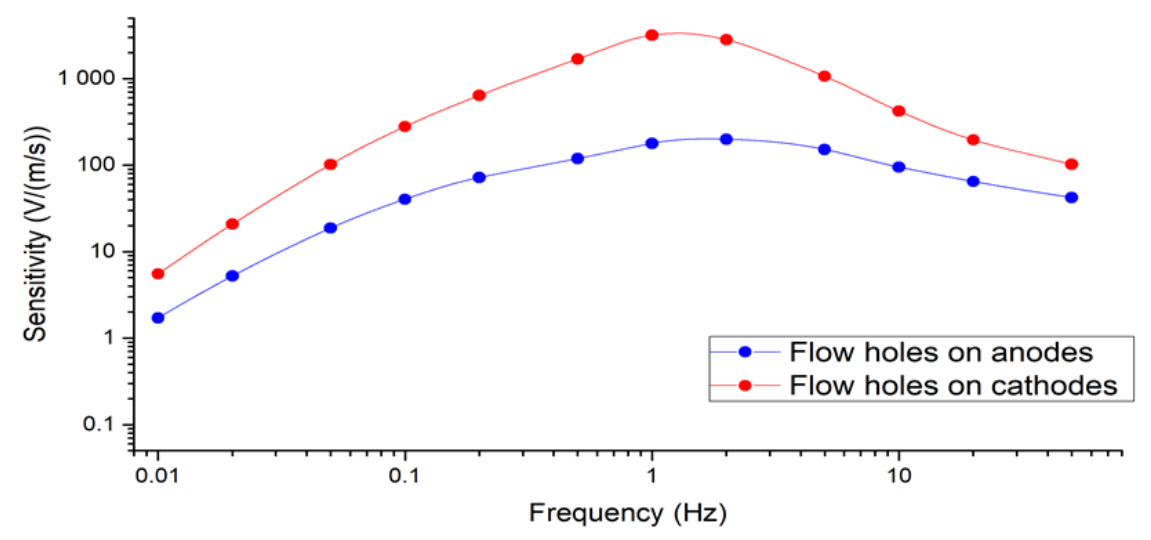

Figure 2. (a) The two-dimensional simulation model of the proposed MEMS-based electrochemical seismometers with two pairs of electrodes integrated on one chip in COMSOL Multiphysics, mainly consisting of the sensing unit, two elastic membranes, and the electrolyte. The simulation models of the sensing units with cathodes, anodes, and numerous flow holes distributed on cathodes (b) or anodes (c), respectively. (d) The enlarged simulation model of the sensing unit with flow holes on cathodes, where $\mathrm{L}_{1}$ represents the thickness of the insulating layer between the anode and the cathode, $\mathrm{L}_{2}$ represents both the thickness of the insulating layer between two pairs of anodes and cathodes and the length of the flow holes at the same time, and $\mathrm{L}_{3}$ represents the diameter of the flow holes. (e) Simulation results (sensitivity vs. input frequency of the volume force) of proposed seismometers with flow holes distributed on anodes or cathodes. 


\section{Fabrication}

The proposed microelectromechanical system-based electrochemical seismometers with two pairs of electrodes integrated on one chip were based on conventional microfabrication including key steps of metal deposition, photolithography, and deep reactive ion etching (DRIE) (see Figure 3).

(a) $\mathrm{SiO}_{2}$ layers $(1 \mu \mathrm{m})$ for insulating were deposited on both sides of the wafer by wet oxidation after wafers were placed in boiled sulfuric acid and deionized water for thorough cleaning.

(b) At first, the pattern of the photoresist mask (AZ1500 photoresist) was prepared on one side of the substrate by photolithography, followed by the cleaning step based on oxygen plasma ( $3 \mathrm{~min})$. Ti $(40 \mathrm{~nm})$ and platinum $\mathrm{Pt}(200 \mathrm{~nm})$ were sputtered in sequence on the patterned surface of the $\mathrm{SiO}_{2}$ film after oxygen plasma (30 s). Subsequently, the anode was formed by lift-off technology where acetone, alcohol, and deionized water were used in turn to remove the underneath photoresist. Finally, the other side of the substrate was processed in the same way, producing symmetrical anodes on both sides of the silicon wafer.

(c) For protecting the surface of anode, the photoresist mask pattern (AZ4620 photoresist) was formed on one side of the substrate by photolithography. Then, about $800 \mathrm{~nm}$ of the $\mathrm{SiO}_{2}$ layer was removed by trifluoromethane etching to form flow holes. Afterwards, the photoresist mask was removed. In the end, the other side was fabricated with the same steps where the concave holes were distributed symmetrically on both $\mathrm{SiO}_{2}$ layers.

(d) Firstly, the pattern of cathodes was transferred from the mask to the surface of $\mathrm{SiO}_{2}$ layers by photolithography (AZ1500 photoresist). Then Ti $(40 \mathrm{~nm})$ and Pt (200 nm) were sputtered successively on the surface of the $\mathrm{SiO}_{2}$ film. Subsequently, the cathodes were formed by lift-off technology. Finally, the other side of the substrate was processed in the same way, generating symmetrical cathodes on both sides of the silicon wafer.

(e) The pattern of the photoresist mask (AZ4620 photoresist) was formed on one side of the silicon wafer. Next, the remaining $\mathrm{SiO}_{2}$ layer was etched by trifluoromethane from the front side. Then, the silicon layer was removed completely by DRIE. Finally, the $\mathrm{SiO}_{2}$ layer on the other side was removed by trifluoromethane etching and then the patterned wafer was cleaned up.

(f) The patterned four electrodes were connected to four pads on two printed circuit boards respectively by wire bonding leveraging Au wires.

(a)

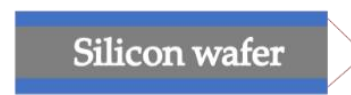

$\mathrm{SiO}_{2}(\mathrm{~d})$

(d)

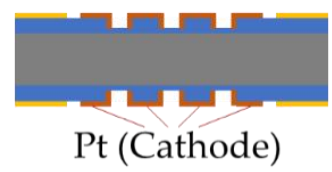

(e)

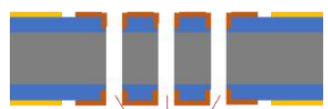

Flow holes (g)

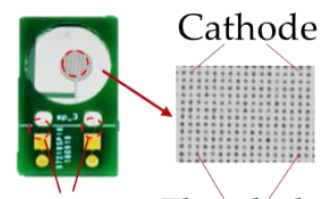

Au wires Flow holes (b)

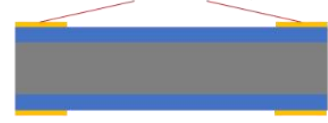

(c)

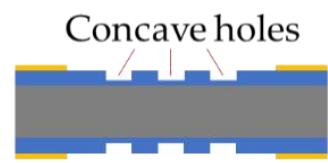

(f)

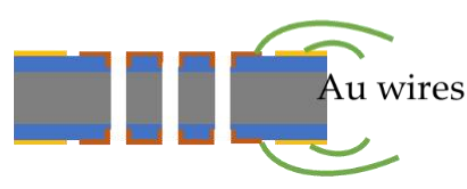

(h)

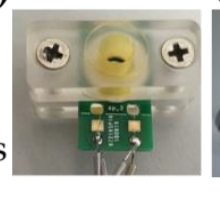

(i)

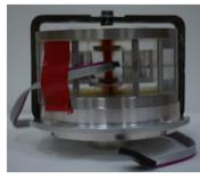

Figure 3. (a-f) The fabrication process of the sensing unit with two pairs of electrodes integrated on one chip, including key steps of (a) deposition of $\mathrm{SiO}_{2}$ layers, (b) deposition and patterning of anodes, (c) formation of concave holes in $\mathrm{SiO}_{2}$ layers, (d) deposition and patterning of cathodes, (e) formation of flow holes on cathodes, and (f) connection with Au wires. Pictures of (g) a fabricated sensing unit,

(h) the assembled sensing unit, and (i) the assembled electrochemical seismometer.

Figure $3 \mathrm{~g}$ shows a fabricated sensing unit, where the dimensions of the sensing unit before and after wire bonding were $12 \mathrm{~mm}$ by $15 \mathrm{~mm}$ and $12 \mathrm{~mm}$ by $20 \mathrm{~mm}$, respectively. Figure 3h shows an 
assembled sensing unit, where an O-ring and an organic glass elements were placed on each side of a fabricated sensing unit, and the screws at both ends of the organic glass were tightened. In addition, a picture of a fabricated cathode with hundreds of flow holes (diameters of $100 \mu \mathrm{m}$ ) was also included in Figure 3g. Figure $3 \mathrm{~h}$ shows the prototype of an assembled electrochemical seismometer, including key components of two elastic membranes, a plexiglass shell, and an assembled sensing unit, which were sealed together by mechanical compression.

In this study, all the steps mentioned above belong to the mature silicon-based fabrication technology. In addition, both the silicon dioxide layers fabricated by wet oxidation and the electrode layers manufactured by sputtering on the silica layer are stable enough. Thus, the seismometers developed in this study are featured with high fabrication repeatability

\section{Devices Characterizations}

In order to compare the performances of the developed seismometers, a commercially available electrochemical seismometer CME6011 was used as a reference. Moreover, comparisons of the proposed seismometers with holes distributed on anodes or cathodes were conducted to verify the simulation results.

\subsection{Amplitude-Frequency Response}

The experiments to characterize amplitude-frequency responses of electrochemical seismometers were conducted on an ultra-low-frequency vibration table (National Institute of Metrology), where the sinusoidal voltage generated by a waveform generator and amplified by a power amplifier was converted to the input velocity. In addition, the voltage-current conversion resistors were set to $1 \mathrm{~K} \Omega$. Figure 4 shows the sensitivities of the proposed seismometers with holes on cathodes or anodes and CME6011, which were quantified as $521.28 \mathrm{~V} /(\mathrm{m} / \mathrm{s})$ vs. $1.59 \mathrm{~V} /(\mathrm{m} / \mathrm{s}) \mathrm{vs} .68 .05 \mathrm{~V} /(\mathrm{m} / \mathrm{s})$ at $0.1 \mathrm{~Hz}$, $3555.57 \mathrm{~V} /(\mathrm{m} / \mathrm{s})$ vs. $7.16 \mathrm{~V} /(\mathrm{m} / \mathrm{s})$ vs. $163.13 \mathrm{~V} /(\mathrm{m} / \mathrm{s})$ at $1 \mathrm{~Hz}$, and $1369.22 \mathrm{~V} /(\mathrm{m} / \mathrm{s})$ vs. $12.14 \mathrm{~V} /(\mathrm{m} / \mathrm{s})$ vs. $162.03 \mathrm{~V} /(\mathrm{m} / \mathrm{s})$ at $10 \mathrm{~Hz}$, respectively. Based on the experimental results, the sensitivities of the proposed seismometer with flow holes on cathodes were more than two orders higher than the counterpart with flow holes on anodes at $1 \mathrm{~Hz}$, which was one order higher than the simulation results. However, the trend of amplitude-frequency responses of the proposed seismometers with flow holes on cathodes or the anodes was consistent with the simulation results. That was because two-dimensional simulations can only provide a qualitative rather than a quantitative estimation of device performances.

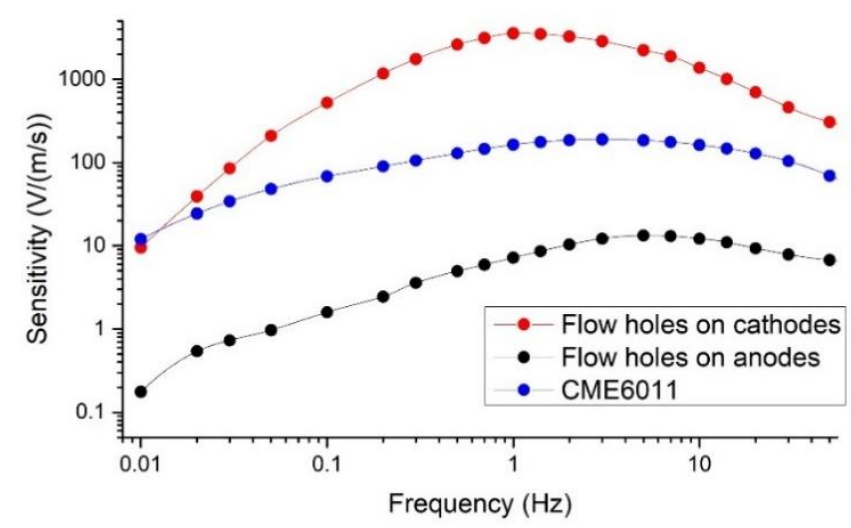

Figure 4. The sensitivities of the proposed electrochemical seismometers with holes on cathodes or anodes and CME6011 as a function of the frequency of the volume force.

In addition, the sensitivities of the proposed seismometer with flow holes on cathodes were significantly higher than the counterpart with flow holes on anodes as a result of faster reduction reaction on cathodes. When the seismometer was subjected to vibration, the electrolyte solution would move in the direction of the flow holes. Therefore, for seismometers with flow holes distributed on 
the anode, most of the products (tri-iodide ions) of the anodic reaction would be taken away by the solution through the flow holes and seldom flowed to the cathode. As for seismometers with flow holes distributed on the cathode, most of the products of the anodic reaction would flow to the flow holes and the cathodes. At the same time, tri-iodide ions in the cathodic reaction are mainly the products of the anodic reaction due to low concentration of tri-iodide ions in electrolyte. Therefore, the concentration of tri-iodide ions around two cathodes was much higher than the counterparts with holes on anodes, leading to faster reduction reaction and higher sensitivities.

As shown in Figure 4, in comparison to CME6011, the sensitivities of the proposed electrochemical seismometer with flow holes on cathodes were comparable at the low-frequency domain, more than one order higher at the intermediate-frequency domain and about five times higher at the high-frequency domain. That was because of enlarged electrode areas and decreased thickness of insulating layers, resulting in an increase of differential tri-iodide ions flux. These results validated the functionality of the proposed electrochemical seismometers in this study. However, the $3 \mathrm{~dB}$ working bandwidth (the frequency range where the amplitude is higher than 0.707 times of the maximum) of the proposed electrochemical seismometer with flow holes on cathodes was narrower than that of CME6011 and that of the proposed electrochemical seismometer with flow holes on anodes. Thus, it is necessary to balance between the sensitivities and the $3 \mathrm{~dB}$ working bandwidth in various applications.

\subsection{Self-Noise Level}

In order to further characterize the self-noise level of the proposed electrochemical seismometer with flow holes on cathodes, it was placed side by side with CME6011 to collect self-noise data. Figure 5 shows the self-noise spectrum densities of both the proposed electrochemical seismometer with flow holes on cathodes and CME6011. Based on these results shown in Figure 5, the noise spectrum densities of the proposed seismometer and CME6011 were quantified as $-139.75 \mathrm{~dB}(101 \mathrm{~g}(\mathrm{~m} / \mathrm{s}) / \mathrm{Hz} 1 / 2)$ vs. $-136.33 \mathrm{~dB}$ at $0.1 \mathrm{~Hz},-161.28 \mathrm{~dB}$ vs. $-159.85 \mathrm{~dB}$ at $1 \mathrm{~Hz}$, and $-134.96 \mathrm{~dB}$ vs. $-127.35 \mathrm{~dB}$ at $10 \mathrm{~Hz}$, respectively. Comparable noise levels of the proposed electrochemical seismometer with flow holes on cathodes and CME6011 were located, validating the functionality of the proposed electrochemical seismometer in this study.

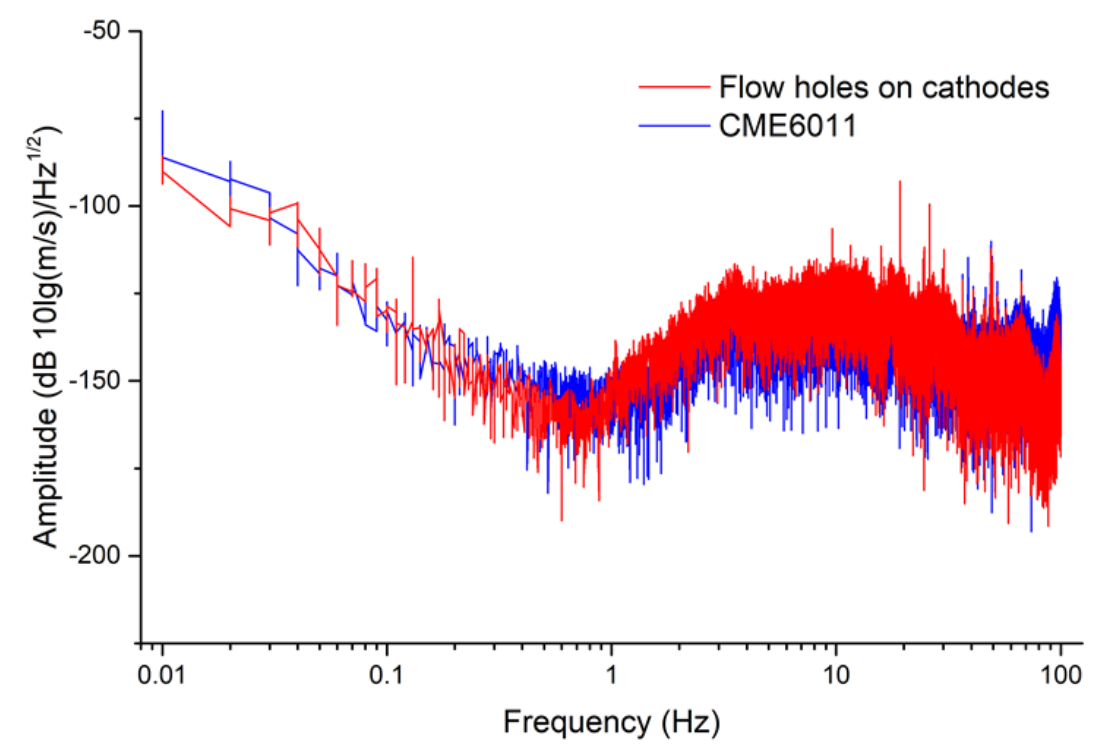

Figure 5. Self-noise spectrum densities of both the proposed electrochemical seismometer with flow holes on cathodes and CME6011. 


\subsection{Transient Response}

Figure 6a shows the transient responses of both the proposed electrochemical seismometer and CME6011 with two events of ground motions, including a weak signal (138-144 s) and a strong signal (330-400 s). Figure $6 \mathrm{~b}$ shows the details of recorded strong signals from $380 \mathrm{~s}$ to $385 \mathrm{~s}$, where the frequency of output voltage was about $7 \mathrm{~Hz}$. Based on these results shown in Figure $6 \mathrm{~b}$, the output voltage amplitudes of these two types of seismometers were quantified as $0.034 \mathrm{~V}$ vs. $0.0031 \mathrm{~V}$ $(381.72 \mathrm{~s})$ and $0.046 \mathrm{~V}$ vs. $0.0039 \mathrm{~V}(384.55 \mathrm{~s})$, demonstrating that the transient responses of the proposed seismometers with holes on cathodes were about 10.97 times and 11.79 times those of CME6011, which was consistent with the test results in amplitude-frequency response. In addition, the correlation coefficient of these two types of electrochemical seismometers was quantified as 0.979 after normalization of output voltage, further validating the functionality of the proposed electrochemical seismometer in this study.
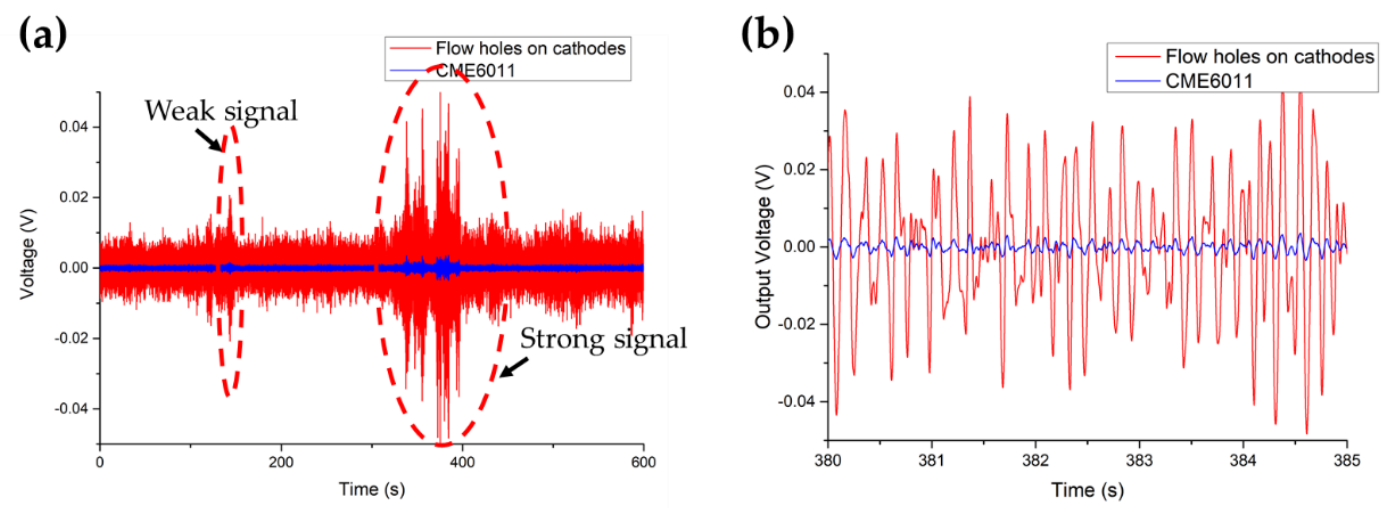

Figure 6. (a) The time-domain responses of both the proposed electrochemical seismometer with flow holes on cathodes and CME6011 with two events of ground motions, including a weak signal (138-144 s) and a strong signal (330-400 s) with details of recorded signals from $380 \mathrm{~s}$ to $385 \mathrm{~s}$ shown in (b).

\section{Conclusion}

This paper demonstrated MEMS based electrochemical seismometers with two pairs of electrodes integrated on a chip. Both simulation and experimental results showed that the sensitivities of the proposed seismometer with flow holes on cathodes were one to two orders higher than the counterpart with flow holes on anodes. In addition, compared to CME6011, the proposed seismometer with flow holes on cathodes indicated significantly higher sensitivities and comparable noise levels. These results validated the functionality of the proposed electrochemical seismometers, which may contribute to seismic monitoring in the near future.

Author Contributions: Conceptualization, X.Z., D.C., and J.W.; data curation, X.Z.; formal analysis, X.Z., C.X., W.Q., and B.L.; funding acquisition, D.C. and J.W.; methodology, X.Z.; project administration, D.C. and J.W.; software, X.Z.; validation, X.Z.; writing—original draft, X.Z.; writing—review and editing, D.C. and J.C.

Funding: This work was supported by the Strategic Priority Research Program of the Chinese Academy of Sciences (Grant No. XDA22020302), the State's Key Project of Research and Development Plan (Grant No. 2016YFC0301801), the National Key Scientific Instrument and Equipment Development Project of China (Grant No. 2013 YQ12035703 and the National Science Fund for Distinguished Young Scholars (Grant No. 61825107).

Conflicts of Interest: The authors declare no conflict of interest. 


\section{References}

1. Havskov, J.; Alguacil, G. Instrumentation in Earthquake Seismology; Springer: Dordrecht, The Netherlands, 2010; pp. 1-5.

2. Niu, F.; Silver, P.G.; Daley, T.M.; Cheng, X.; Majer, E.L. Preseismic velocity changes observed from active source monitoring at the Parkfield SAFOD drill site. Nature 2008, 454, 204-208. [CrossRef] [PubMed]

3. DeFreitas, J.M. Recent developments in seismic seabed oil reservoir monitoring applications using fibre-optic sensing networks. Meas. Sci. Technol. 2011, 22, 1-30.

4. Levinzon, F.A. Ultra-Low-Noise Seismic Piezoelectric Accelerometer with Integral FET Amplifier. IEEE Sens. J. 2012, 12, 2262-2268. [CrossRef]

5. Donald, J.M.; Brian, D.M. An Ultra-Low Noise MEMS Accelerometer for Seismic Imaging. In Proceedings of the IEEE Sensors Conference, Limerick, Ireland, 28-31 October 2011; pp. 1281-1284.

6. Kamata, M. Front and fidelity for seismic acquisition. In Proceedings of the 10th SEGJ International Symposium, Kyoto, Japan, 20-22 November 2011; pp. 17-20.

7. Liang, T.C.; Lin, Y.L. Fiber optic sensor for detection of ground vibrations. In Proceedings of the Third Asia Pacific Optical Sensors Conference, Sydney, Australia, 30 January 2012. 835109.

8. Zhang, G.H.; Hu, S.Y. Dynamic characteristics of moving-coil geophone with large damping. Int. J. Appl. Electromagnet Mech. 2010, 33, 565-571. [CrossRef]

9. Agafonov, V.M.; Egorov, E.V.; Zaitsev, D.L. Molecular electronic linear accelerometers. Preliminary test results. Gyroscopy Navig. 2010, 1, 246-251. [CrossRef]

10. Laudati, A.; Mennella, F.; Giordano, M.A. Fiber-Optic Bragg Grating Seismic Sensor. IEEE Photonics Technol. Lett. 2007, 19, 1991-1993. [CrossRef]

11. Huang, H.; Agafonov, V.; Yu, H. Molecular electric transducers as motion sensors: A review. Sensors 2013, 13, 4581-4597. [CrossRef] [PubMed]

12. Krishtop, V.G.; Agafonov, V.M.; Bugaev, A.S. Technological principles of motion parameter transducers based on mass and charge transport in electrochemical microsystems. Russ. J. Electrochem. 2012, 48, 746-755. [CrossRef]

13. Liang, M.B.; Huang, H.; Agafonov, V.; Tang, R. Molecular electronic transducer based planetary seismometer with new fabrication process. In Proceedings of the IEEE 29th International Conference on Micro Electro Mechanical Systems (MEMS), Shanghai, China, 24-28 January 2016; pp. 986-989.

14. Abramovich, I.A.; Kharlamov, A.V. Electrochemical Transducers and a Method for Fabricating the Same. U.S. Patent 6,576,103B2, 10 June 2003.

15. Chen, D.; Li, G.; Wang, J.; Chen, J.; He, W.; Fan, Y.; Deng, T.; Wang, P. A micro electrochemical seismic sensor based on MEMS technologies. Sens. Actuators A 2013, 202, 85-89. [CrossRef]

16. Huang, H.; Carande, B.; Tang, R.; Oiler, J.; Zaitsev, D.; Agafonov, V.; Yu, H. A micro seismometer based on molecular electronic transducer technology for planetary exploration. Appl. Phys. Lett. 2013, 102, 193512. [CrossRef]

17. Deng, T.; Sun, Z.; Li, G.; Chen, J.; Chen, D.; Wang, J. Microelectromechanical system-based electrochemical seismic sensors with an anode and a cathode integrated on one chip. J. Micromech. Microeng. 2017, $27,025004$. [CrossRef]

18. Li, G.; Sun, Z.; Wang, J.; Chen, D.; Chen, J.; Chen, L.; Xu, C.; Qi, W.; Zheng, Y. A Flexible Sensing Unit Manufacturing Method of Electrochemical Seismic Sensor. Sensors 2018, 18, 1165. [CrossRef] [PubMed]

(C) 2019 by the authors. Licensee MDPI, Basel, Switzerland. This article is an open access article distributed under the terms and conditions of the Creative Commons Attribution (CC BY) license (http://creativecommons.org/licenses/by/4.0/). 